University of Nebraska - Lincoln

DigitalCommons@University of Nebraska - Lincoln

John R. Hardy Papers

Research Papers in Physics and Astronomy

3-15-1969

\title{
Lattice Dynamics and Second-Order Raman Spectrum of NaF
}

John R. Hardy

University of Nebraska - Lincoln

A. M. Karo

University of California, Livermore, California

I. W. Morrison

University of California, Livermore, California

C. T. Sennett

Royal Radar Establishment, Great Malvern, Worcestershire, England

J. P. Russell

R.C.A . Laboratories, Zurich, Switzerland

Follow this and additional works at: https://digitalcommons.unl.edu/physicshardy

Part of the Physics Commons

Hardy, John R.; Karo, A. M.; Morrison, I. W.; Sennett, C. T.; and Russell, J. P., "Lattice Dynamics and SecondOrder Raman Spectrum of NaF" (1969). John R. Hardy Papers. 6.

https://digitalcommons.unl.edu/physicshardy/6

This Article is brought to you for free and open access by the Research Papers in Physics and Astronomy at DigitalCommons@University of Nebraska - Lincoln. It has been accepted for inclusion in John R. Hardy Papers by an authorized administrator of DigitalCommons@University of Nebraska - Lincoln. 


\title{
Lattice Dynamics and Second-Order Raman Spectrum of NaF*
}

\author{
J. R. HARDY $\dagger$ \\ Behlen Laboratory of Physics, University of Nebraska, Lincoln, Nebraska 68508 \\ AND \\ A. M. Karo AND I. W. MORRSION \\ Lawrence Radiation Laboratory, University of California, Livermore, California 94550 \\ AND \\ C. T. SennetT \\ Royal Radar Establishment, Great Malvern, Worcestershire, England \\ AND \\ J. P. RUSSELL \\ R.C.A. Laboratories, Zurich, Switzerland \\ (Received 16 May 1968; revised manuscript received 12 September 1968)
}

\begin{abstract}
The second-order Raman spectrum of $\mathrm{NaF}$ has been measured using an argon laser source. For a light beam incident along the [010] direction, polarized along the [001] direction, and viewed along the [100] direction, the scattered radiation is almost completely polarized. It is shown theoretically that it is possible to account for the polarization and the detailed shape of the observed spectrum within the experimental resolution using a Raman polarizability tensor whose second derivatives with respect to nuclear displacements depend only on the positions of first neighbors in the crystal, provided one uses the phonon frequencies derived from a deformation dipole model that includes second-neighbor $F^{-}-F^{-}$interactions. By assuming that the polarizability is affected only by central displacements of the nearest neighbors, the number of independent polarizability derivatives is reduced from eight to three. This assumption also gives a vanishing depolarized intensity in agreement with the experimental results. The second-order difference spectra have also been evaluated, and are found to be much more sensitive to the relative magnitudes of the various polarizability derivatives.
\end{abstract}

\section{INTRODUCTION}

W ITH the recent advent of laser sources there has been a resurgence of interest in Raman spectroscopy, which has consequently progressed from being an experimental tour de force to being a standard spectroscopic technique for the study of the solid state. The reason for this change is that a laser source is both highly intense and highly monochromatic; the first property makes it possible to observe spectra one or two orders of magnitude more rapidly, and the second property, in principle, ensures that the measured spectrum is clearly defined and not obscured by Rayleigh-scattered exciting radiation, as is the case of spectra taken with a conventional mercury discharge source. However, this second property can only be fully exploited if one uses a photon counting detector rather than a photographic plate, since halation about the central line obscures the low-frequency part of the spectrum. Moreover the response of a photographic plate is by no means linear, so that a microdensitometer trace of a photographically recorded spectrum does not provide a true picture of that spectrum.

However, as a first stage, one can simply utilize the great intensity of a laser source to observe Raman spectra which are too weak to be seen by conventional

* Work performed, in part, under the auspices of the U. S. Atomic Energy Commission. t Consultant to Lawrence Radiation Laboratory, Livermore,
Calif.

179 methods, and provided the frequency shifts are large, photographic recording is simple and adequate. Both characteristics are present for the fluorides of the lighter alkali metals since the low polarizabilities of the constituent ions lead to weak Raman scattering, while the small ionic masses lead to high phonon frequencies. Also, since these crystals have the $\mathrm{NaCl}$ structure, the lowest order allowed Raman scattering is a two-phonon process, which is inherently weak.

The most extreme case is $\mathrm{LiF}$, but so far as we know, it has not yet been possible to observe the Raman spectrum of this crystal. The next crystal in the sequence is, of course, $\mathrm{NaF}$. In this case we are fortunate in that the phonon dispersion curves have recently been measured both by thermal diffuse $x$-ray scattering ${ }^{1}$ and by inelastic neutron scattering. ${ }^{2}$ Furthermore, we have shown in another paper ${ }^{3}$ that theoretical calculations based on our deformation dipole model with secondneighbor $F^{-}-F^{-}$interactions reproduce the observed dispersion curves extremely well when one considers that none of the model parameters have been adjusted to fit the observed dispersion curves. Thus, we may reasonably assume that our model provides a satisfactory way of calculating the frequencies of all pairs of phonons involved in second-order Raman processes.

\footnotetext{
$1 \mathrm{~J}$. D. Pirie and T. Smith (private communication).

2 W. J. L. Buyers, Phys. Rev. 153, 923 (1967).

3 A. M. Karo and J. R. Hardy, University of California Lawrence Radiation Laboratory Report No. UCRL-71085 (unpublished).
} 


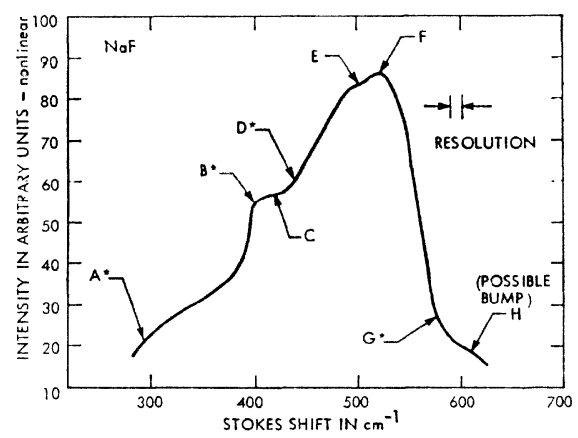

FIG. 1. Experimental $300^{\circ} \mathrm{K}$ second-order Raman spectrum of $\mathrm{NaF}$ (resolution $\approx 10 \mathrm{~cm}^{-1}$ ). The positions of definite features are marked $A, B$, etc. (Asterisks refer to the edges of the main features; the small high-frequency bump $H$ may be a third-order line.)

This degree of certainty about the phonon frequencies allows us to concentrate our attention on the selection rules governing Raman processes. The first and most basic of these, which is imposed by the translational symmetry of the lattice, states that, in a two-phonon Raman process, the phonons involved have to have equal and opposite wave vectors, $\mathbf{q}$ and $-\mathbf{q}$. Subject to this restriction, there are three types of Raman processes: the Stokes process, in which two phonons are created; the anti-Stokes process, in which two phonons are destroyed; and difference processes, in which one phonon is created and one destroyed. The last two types of processes are only present at finite temperatures, since a destruction process is proportional to $N_{\mathrm{q}, j}$, the number of phonons initially present ( $j$ being the branch index), while a creation process is proportional to $N_{\mathrm{q}, j}+1$. Therefore, a second-order Stokes process is proportional to $\left(N_{\mathrm{q}, j}+1\right)\left(N_{-\mathrm{q}, j^{\prime}}+1\right)$, while the corresponding anti-Stokes and difference processes are proportional to $N_{\mathrm{q}, j} N_{-\mathrm{q}, j^{\prime}}$ and $\left(N_{\mathrm{q}, j}+1\right)$ $\times N_{-q, j^{\prime}}$, respectively. At absolute zero, when all the $N$ 's are zero, obviously only the first type of process remains.

With these facts in mind, one can make a first prediction of the expected form of the second-order Raman spectrum by assuming that all possible allowed combinations have the same weights, apart from the differences in phonon occupation numbers. In an earlier paper on $\mathrm{NaCl},{ }^{4}$ we computed the second-order Raman spectrum of that crystal on the basis of this combined density-of-states approximation. The calculation was carried out in the $0^{\circ} \mathrm{K}$ limit, and thus we obtained only the Stokes component. At the time the results seemed to be in reasonable agreement with the experimental spectrum of Welsh et al., ${ }^{5}$ even though this spectrum was taken at $300^{\circ} \mathrm{K}$. However, subsequent measurements by Worlock ${ }^{6}$ taken with a laser source, both at

A. M. Karo and J. R. Hardy, Phys. Rev. 141, 696 (1966).

5 H. L. Welsh, M. F. Crawford, and W. J. Staple, Nature 164, 737 (1949).

o J. Worlock, paper presented at 1966 Edinburgh Conference on Phonons; and (private communication).
300 and $80^{\circ} \mathrm{K}$, are in marked disagreement with the data of Welsh et al..$^{5}$ Moreover, the use of a laser source made it possible to determine the degree of polarization of the Raman-scattered radiation, and it was found that for a beam incident along the [010] direction, polarized along the $[(0) 1]$ direction and viewed along the [100] direction, the scattered radiation was almost completely polarized in the same direction as the incident beam. This indicates that the density-of-states approximation is inadequate, and that it is necessary to consider a detailed model of the Raman polarizability tensor. However, the origin of the discrepancy between Worlock's results and those of Welsh $e t$ al. is not at all clear. One possibility, suggested by Worlock ${ }^{6}$ is that in the conventional experiment, where the specimen is illuminated by a high-pressure mercury discharge lamp its temperature becomes significantly elevated above that of its surroundings. Thus the spectra taken by Welsh et al. are appropriate, not to $300^{\circ} \mathrm{K}$, but to some higher temperature. This is a possibility, but it raises a further problem as to whether or not a change in temperature is capable of radically modifying the general shape as opposed to the intensity of the Stokes component of the spectrum. If this is so, then Worlock's hypothesis is probably true; certainly the difference between his 80 and $300^{\circ} \mathrm{K}$ spectra suggests that such a modification is possible. Therefore, in addition to considering theoretically the effects of variations in the Raman polarizability tensor, it is of importance to carry out our calculations for at least two different temperatures, the most obvious pair being 0 and $300^{\circ} \mathrm{K}$ (i.e., room temperature).

These considerations for $\mathrm{NaCl}$ are equally relevant to the present theoretical and experimental investigation of $\mathrm{NaF}$, which we decided to pursue first before attempting any further work on $\mathrm{NaCl}$.

\section{EXPERIMENTAL RESULTS}

The apparatus used has been described in detail elsewhere. ${ }^{7}$ The source was a 1-W Raytheon argon laser, and the spectra were recorded photographically with a Hilger and Watts Raman spectrograph. The specimen was a high optical quality $\mathrm{NaF}$ crystal, cubic in shape, having $\{100\}$ faces. The scattering geometry was the same as that used by Worlock ${ }^{6}$ for $\mathrm{NaCl}$. The use of exposures up to $6 \mathrm{~h}$ made it possible to observe the Raman-scattered Stokes component from both the $4880 \AA$ and the $5145 \AA$ exciting lines. The polarized component alone was observable, implying that the depolarized component was at least five times weaker, and thus one can say that the scattered radiation is almost completely polarized.

The spectrum obtained is shown in Fig. 1, and all clearly defined features have been labeled. It should be stressed that the intensity scale, which refers to a

${ }^{7}$ J. P. Russell, in Phonons, edited by R. W. H. Stevenson (Plenum Press, Inc., New York, 1966), p. 235. 
Fig. 2. Plots of predicted spectral intensity against frequency shift from the exciting line (intensity in arbitrary units but on a linear scale) for the rigid ion (RI) model assuming constant polarizability matrix elements. (a) $300^{\circ} \mathrm{K}$ Stokes and anti-Stokes spectra; positions of experimental features (see Fig. 1) are indicated by $A, B$, etc. Features marked $(P)$ etc., are definite theoretical features which are absent from the observed spectrum. (b) Corresponding $300^{\circ} \mathrm{K}$ RI difference spectrum. (c) $0^{\circ} \mathrm{K}$ Stokes spectrum with corresponding combination frequencies plotted against wave vector $\mathbf{q}$ along various symmetry directions. The features labeled $a, b$, etc., on the spectrum correspond to the appropriately marked critical points on the combined dispersion curve. Any given feature may have more than one critical point associated with it. (Note: Although we are comparing the theoretical $300^{\circ} \mathrm{K}$ spectrum with the observed spectrum, it should be remembered that in the present approximation, the theoretical spectrum is completely depolarized.)
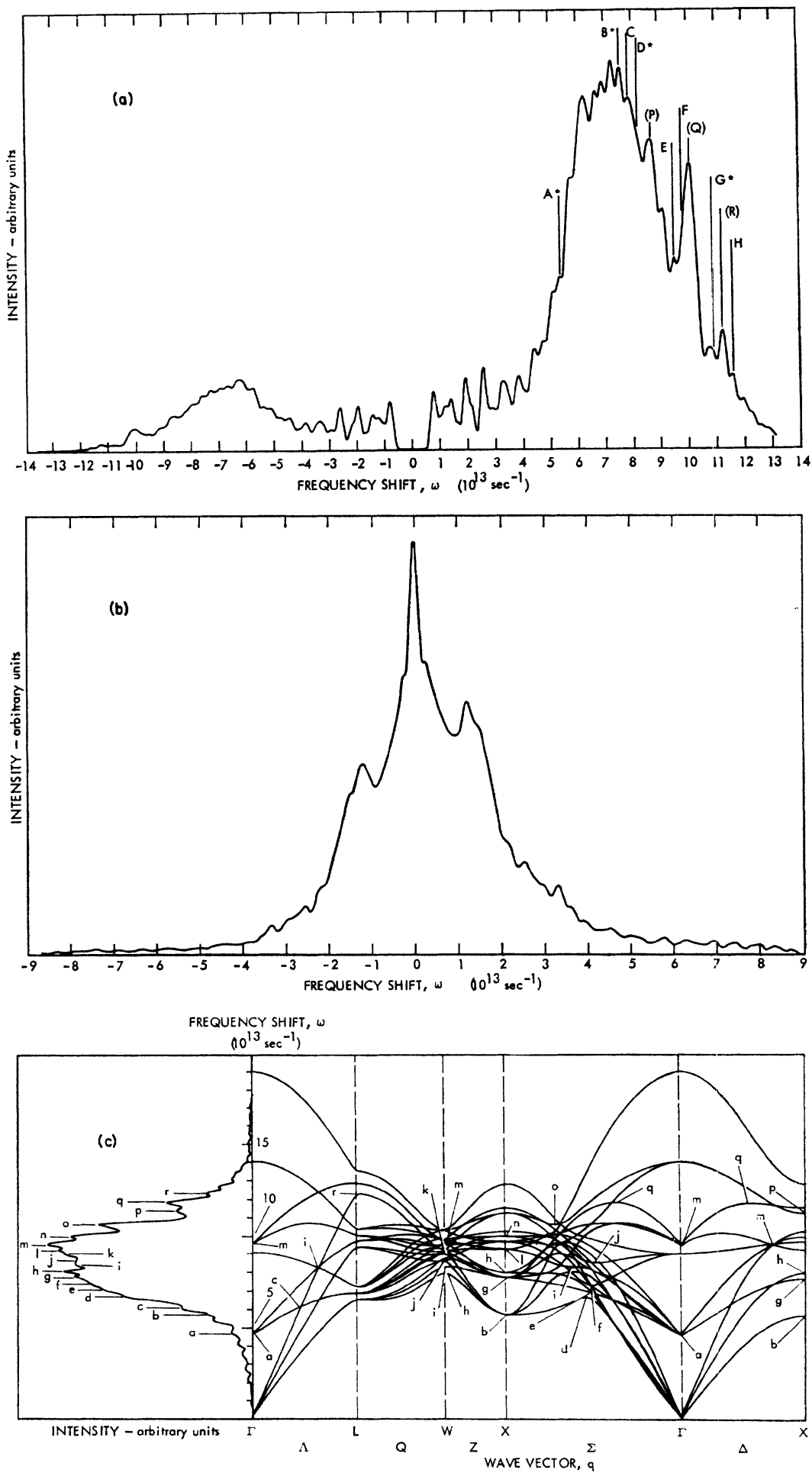

microdensitometer trace of the photographically recorded spectrum, is not linear, since the response of photographic plates is both nonlinear at any given wavelength and also wavelength dependent. However, the relative intensities of various features are qualitatively correct; i.e., where one feature appears weaker than another in the observed trace, then it is also weaker in absolute intensity. 


\section{COMBINED DENSITY OF STATES}

The first stage in our interpretation of the observed second-order Raman spectrum is the computation of a simple combined density of states, without selection rules but including temperature weighting.

Thus, we compute a density function $\rho(\omega)$ given by

$$
\begin{aligned}
\rho(\omega)=\frac{1}{\Delta \omega} \sum_{q, j^{\prime}} \int_{\omega}^{\omega+\Delta \omega} d \omega\left(N_{\mathrm{q}, j}+1\right)\left(N_{-\mathrm{q}, j^{\prime}}+1\right) \\
\times \delta\left(\omega-\omega_{\mathrm{q}, j^{-}}-\omega_{-\mathrm{q}, j^{\prime}}\right)+\left(N_{\mathrm{q}, j}+1\right)\left(N_{-\mathrm{q}, j^{\prime}}\right) \\
\times \delta\left(\omega-\omega_{\mathrm{q}, j}+\omega_{-\mathrm{q}, j^{\prime}}\right)+\left(N_{\mathrm{q}, j}\right)\left(N_{-\mathrm{q}, j^{\prime}}\right) \\
\times \delta\left(\omega+\omega_{\mathrm{q}, j}+\omega_{-\mathrm{q}, j^{\prime}}\right),
\end{aligned}
$$

where the $N$ 's are the phonon occupation numbers appropriate to the temperature being considered. In practice, we compute a histogram of $\rho(\omega)$ obtained using a finite sample of $\mathbf{q}$ vectors. In the process we deliberately make $\Delta \omega$ too small. Then, to the irregular histogram steps so obtained, we fit a sequence of Gaussians and adjust their mutual half-widths until a smooth curve is obtained. We have used this technique in earlier papers ${ }^{8,9}$ and found that the curves which resulted reveal considerably more true detail than simple histograms.

Together with the combined densities of states we also compute combined dispersion curves (i.e., $\omega=\omega_{q, j}+\omega_{-q, j^{\prime}}$ versus $\left.\mathbf{q}\right)$, which are plotted on the same frequency scale as the combined density of states. These curves have been obtained for all the principal symmetry directions of the $\mathrm{NaCl}$ structure, since whenever one of these curves has zero slope or wherever two such curves cross, we expect a Van Hove singularity ${ }^{10,11}$ in the density of states. Also, for convenience and clarity, we have obtained separate plots of the difference spectra, given by the second term in Eq. (1).

Thus, in Fig. 2 we show the combined densities of states for the rigid ion (RI) model of $\mathrm{NaF}$ which has been described in detail elsewhere. ${ }^{3}$ The 300 and $0^{\circ} \mathrm{K}$ results are shown separately. For the room-temperature case the difference bands are also obtained; and for the low-temperature case, where the anti-Stokes and difference bands are absent, the combined dispersion curves are shown. In Fig. 3 we show the corresponding results for the deformation-dipole next-nearest-neighbor (DDNNN) model, which we have found ${ }^{3}$ gives the closest fit to the observed single-phonon dispersion curves. ${ }^{1,2}$

For both models, the basic histogram is derived for a sample of $8000 \mathrm{q}$ vectors within the first Brillouin zone. We believe that our Gaussian-smoothing technique makes this equivalent in the amount of detail revealed

\footnotetext{
8 A. M. Karo and J. R. Hardy, Phys. Rev. 160, 702 (1967).

A. M. Karo and J. R. Hardy, J. Chem. Phys. 48, 3173 (1968).

${ }^{10}$ L. Van Hove, Phys. Rev. 89, 1189 (1953).

1 J. C. Phillips, Phys. Rev. 104, 1263 (1956).
}

to a simple histogram derived for a sample of $64000 \mathrm{q}$ vectors in the reduced zone.

In considering the two room-temperature combined densities of states, it is seen that the DDNNN model reproduces the observed spectrum somewhat better, with one important discrepancy: The sharp subsidiary peak marked " $M$ " on the theoretical spectrum is completely absent from the observed spectrum. Also, there is a less obvious discrepancy in that the strong subsidiary peak " $L$ " on the low-frequency side of the main peak in the theoretical combined density of states is suppressed in the observed spectrum. Furthermore, the observed major peak " $F$ " occurs at a significantly higher frequency that in the theoretical combined density of states. However, the theoretical rigid ion results can be seen to be significantly worse; in particular, there are three unobserved high-frequency peaks, " $P$," " $Q$," and " $R$," and the broad main peak is even further below the position of the observed peak.

Unfortunately, not very much can be said about the difference spectra since theoretically these are relatively weak, and the computed bands occur at frequencies too close to the exciting radiation to be observed photographically. This is to be regretted, for it is evident from both Figs. 2 and 3 that the difference spectra are somewhat more sensitive to the model one uses than are the Stokes and anti-Stokes spectra. Moreover, this is found to be even more apparent in our later calculations.

It is evident from comparison of the Stokes spectra alone that the combined density-of-states approximation is inadequate. Furthermore, this approximation does nothing to explain the polarization of the observed spectrum. To proceed further, it is evidently necessary to consider a detailed model of the polarizability tensor, and this we shall now proceed to do.

\section{DERIVATION OF THE RAMAN POLARIZABILITY TENSOR}

In principle, the second-order Raman polarizability tensor is calculated by applying fourth-order perturbation theory, where one considers those terms that are second order in both the electron-photon and the electron-phonon interactions. This is similar to the calculation of Loudon $^{12}$ for the first-order Raman scattering in diamond-structure materials. However, there one need consider only terms that are linear in the electron-phonon interaction. For the second-order tensor, such computation is at present totally impractical since it requires a detailed knowledge of the band structure of the crystal and of the electron-photon and electron-phonon matrix elements throughout the Brillouin zone. Knowledge of these quantities, particularly the electron-phonon matrix elements, is far too incomplete for these purposes.

\footnotetext{
12 R. Loudon, Proc. Roy. Soc. (London) A275, 218 (1963).
} 
Fig. 3. Results, as given in Fig. 2, for the DDNNN model. (a) $300^{\circ} \mathrm{K}$ Stokes and anti-Stokes spectra with experimental features as in Fig. 2. (b) Difference bands at $300^{\circ} \mathrm{K}$. (c) $0^{\circ} \mathrm{K}$ Stokes spectrum versus combined dispersion curves with critical points indicated on both.
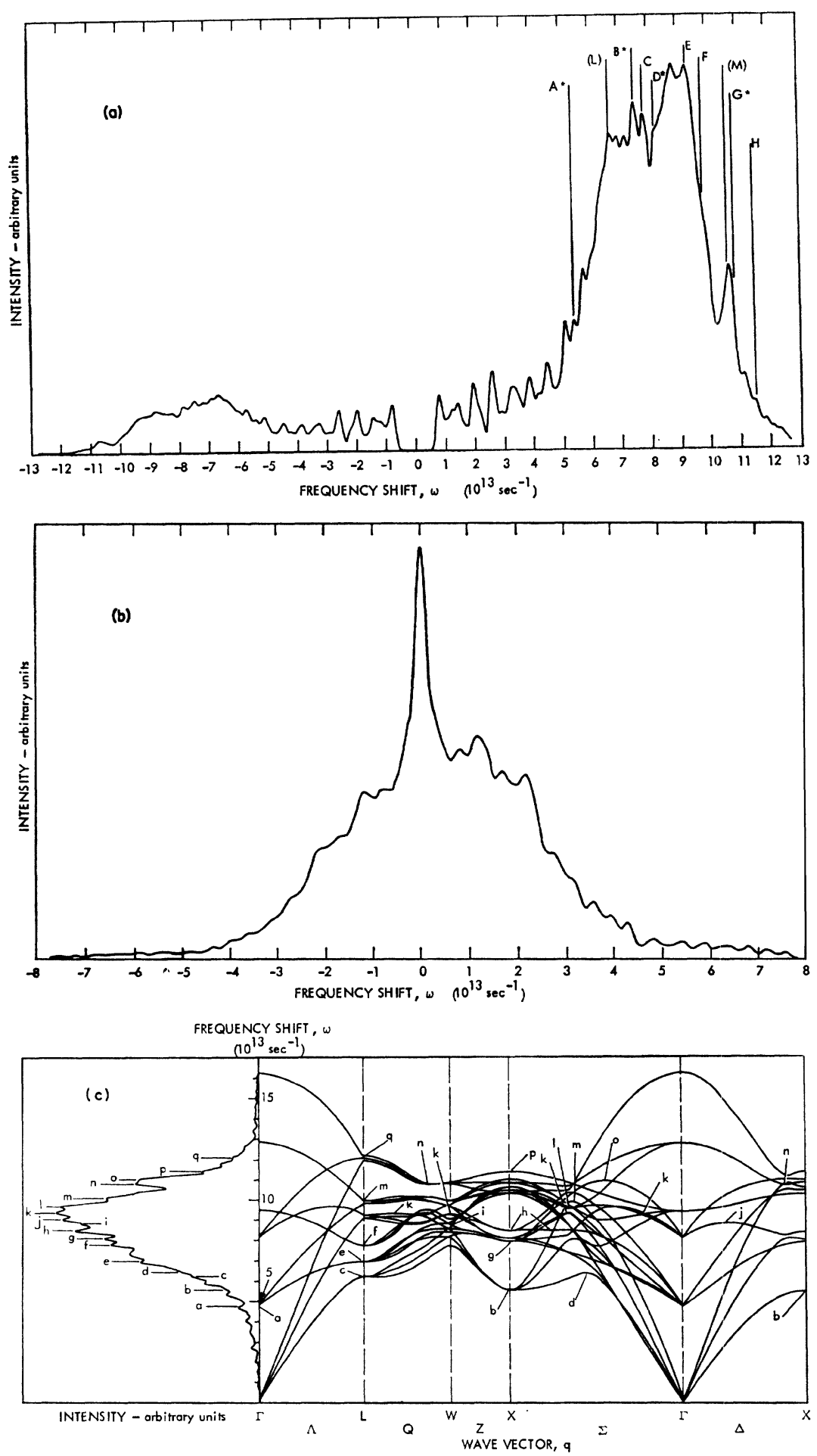

We are thus compelled to fall back on the phenomenological theory of Born and Huang ${ }^{13}$ based on making a power-series expansion of the electronic polarizability in the nuclear displacements.

${ }^{18}$ M. Born and K. Huang, Dynamical Theory of Crystal Lattices (Oxford University Press, New York, 1954), p. 306.
Therefore, if $P_{\alpha \beta}$ is the polarizability, we may write

$$
P_{\alpha \beta}=P_{\alpha \beta}{ }^{0}+\sum_{\substack{l l^{\prime} \\ k k^{\prime} \\ \gamma \delta}} P_{\alpha \beta, \gamma \delta}\left(l k, l^{\prime} k^{\prime}\right) \xi(l, k)_{\gamma} \xi\left(l^{\prime}, k^{\prime}\right)_{\delta},
$$

where we follow the usual notation: Greek subscripts 
refer to Cartesian components; $l, l^{\prime}$ are cell indices; and $k, k^{\prime}$ are either 1 or 2 , according to the type of ion considered. The $\xi$ 's are nuclear displacements and $P_{\alpha \beta, \gamma \delta}\left(l k, l^{\prime} k^{\prime}\right)$ is the second derivative of $P_{\alpha \beta}$ with respect to $\xi(l, k)_{\gamma}$ and $\xi\left(l^{\prime}, k^{\prime}\right)_{\delta}$. (We note that the corresponding first derivatives are zero by $s !$ mmetry.)

The next step is to Fourier transform $P_{\alpha \beta}$. Before proceeding to do this, it is in order to observe that this phenomenological approach is unlikely to be of much practical value unless it is possible to restrict the summation over pairs of lattice sites in Eq. (2) to near neighbors. Otherwise, the number of unknown parameters will be far too large to be fixed in any meaningful way. In practice, we are in immediate trouble if we go only as far as first neighbors, since even for these, symmetry alone will not reduce the number of distinct components of $P_{\alpha \beta, \gamma \delta}\left(l k, l^{\prime} k^{\prime}\right)$ below eight, and we shall need additional information to reduce this number further. Fortunately, as we shall see in detail later, the strong polarization of the observed spectrum is extremely helpful in this respect. It shall be our purpose to show that this reduces the number of parameters to the point where the shape of the calculated spectrum is reasonably insensitive to the relative magnitudes of the remaining parameters.

For the scattering geometry of interest to us, only the two polarizability elements $P_{z z}$ and $P_{y z}$ are required. When the second term in Eq. (2) is Fourier transformed, the terms which couple to phonon pairs of wave vectors $\mathbf{q}$ and $-\mathbf{q}$ and branch indices $j$ and $j^{\prime}$, i.e.,

$$
P_{z z}\left(\mathbf{q}, j ;-\mathbf{q}, j^{\prime}\right) \text { and } P_{y z}\left(\mathbf{q}, j ;-\mathbf{q}, j^{\prime}\right),
$$

are given by

$$
\begin{aligned}
P_{z z}\left(\mathbf{q}, j ;-\mathbf{q}, j^{\prime}\right)= & \left\{[a+2 b]\left[\sigma_{z}(k \mid \mathbf{q}, j) \sigma_{z}\left(k \mid-\mathbf{q}, j^{\prime}\right)+\sigma_{z}\left(k^{\prime} \mid \mathbf{q}, j\right) \sigma_{z}\left(k^{\prime} \mid-\mathbf{q}, j^{\prime}\right)\right]+\left[c+d_{1}+d_{2}\right]\right. \\
& \times\left[\sigma_{x}(k \mid \mathbf{q}, j) \sigma_{x}\left(k \mid-\mathbf{q}, j^{\prime}\right)+\sigma_{x}\left(k^{\prime} \mid \mathbf{q}, j\right) \sigma_{x}\left(k^{\prime} \mid-\mathbf{q}, j^{\prime}\right)+\sigma_{y}(k \mid \mathbf{q}, j) \sigma_{y}\left(k \mid-\mathbf{q}, j^{\prime}\right)\right. \\
& \left.\left.+\sigma_{y}\left(k^{\prime} \mid \mathbf{q}, j\right) \sigma_{y}\left(k^{\prime} \mid-\mathbf{q}, j^{\prime}\right)\right]\right\}-\left\{\left[\sigma_{z}\left(k^{\prime} \mid \mathbf{q}, j\right) \sigma_{z}\left(k \mid-\mathbf{q}, j^{\prime}\right)+\sigma_{z}(k \mid \mathbf{q}, j) \sigma_{z}\left(k^{\prime} \mid-\mathbf{q}, j^{\prime}\right)\right]\right. \\
& \times\left[a \cos \left(q_{z} r_{0}\right)+b\left(\cos \left(q_{x} \boldsymbol{r}_{0}\right)+\cos \left(q_{y} \boldsymbol{r}_{0}\right)\right)\right]+\left[\sigma_{x}\left(k^{\prime} \mid \mathbf{q}, j\right) \sigma_{x}\left(k \mid-\mathbf{q}, j^{\prime}\right)+\sigma_{x}(k \mid \mathbf{q}, j) \sigma_{x}\left(k^{\prime} \mid-\mathbf{q}, j^{\prime}\right)\right] \\
& \times\left[d_{2} \cos \left(q_{x} r_{0}\right)+c \cos \left(q_{y} \boldsymbol{r}_{0}\right)+d_{1} \cos \left(q_{z} \boldsymbol{r}_{0}\right)\right]+\left[\sigma_{y}\left(k^{\prime} \mid \mathbf{q}, j\right) \sigma_{y}\left(k \mid-\mathbf{q}, j^{\prime}\right)+\sigma_{y}(k \mid \mathbf{q}, j)\right. \\
& \left.\left.\quad \times \sigma_{y}\left(k^{\prime} \mid-\mathbf{q}, j^{\prime}\right)\right]\left[c \cos \left(q_{x} \boldsymbol{r}_{0}\right)+d_{2} \cos \left(q_{y} r_{0}\right)+d_{1} \cos \left(q_{z} \boldsymbol{r}_{0}\right)\right]\right\}, \quad(3)
\end{aligned}
$$

$$
\begin{aligned}
P_{y z}\left(\mathbf{q}, j ;-\mathbf{q}, j^{\prime}\right)= & {[e+f+g]\left[\sigma_{y}(k \mid \mathbf{q}, j) \sigma_{z}\left(k \mid-\mathbf{q}, j^{\prime}\right)+\sigma_{z}(k \mid \mathbf{q}, j) \sigma_{y}\left(k \mid-\mathbf{q}, j^{\prime}\right)+\sigma_{y}\left(k^{\prime} \mid \mathbf{q}, j\right) \sigma_{z}\left(k^{\prime} \mid-\mathbf{q}, j^{\prime}\right)\right.} \\
& \left.+\sigma_{z}\left(k^{\prime} \mid \mathbf{q}, j\right) \sigma_{y}\left(k^{\prime} \mid-\mathbf{q}, j^{\prime}\right)\right]-\left\{\left[\sigma_{y}\left(k^{\prime} \mid \mathbf{q}, j\right) \sigma_{z}\left(k \mid-\mathbf{q}, j^{\prime}\right)+\sigma_{y}(k \mid \mathbf{q}, j) \sigma_{z}\left(k^{\prime} \mid-\mathbf{q}, j^{\prime}\right)\right]\right. \\
& \times\left[e \cos \left(q_{x} r_{0}\right)+f \cos \left(q_{y} \boldsymbol{r}_{0}\right)+g \cos \left(q_{z} r_{0}\right)\right]+\left[\sigma_{z}\left(k^{\prime} \mid \mathbf{q}, j\right) \sigma_{y}\left(k \mid-\mathbf{q}, j^{\prime}\right)+\sigma_{z}(k \mid \mathbf{q}, j)\right. \\
& \left.\left.\times \sigma_{y}\left(k^{\prime} \mid-\mathbf{q}, j^{\prime}\right)\right]\left[e \cos \left(q_{x} \boldsymbol{r}_{0}\right)+f \cos \left(q_{z} \boldsymbol{r}_{0}\right)+g \cos \left(q_{y} \boldsymbol{r}_{0}\right)\right]\right\} .
\end{aligned}
$$

These are the general expressions when the summation over lattice sites in the second term on the right-hand side of Eq. (2) is restricted to first neighbors (cf. Born and Bradburn ${ }^{14}$ ).

The eight constants $a, b, \cdots, g$ are the eight independent polarizability derivatives, $r_{0}$ is the nearestneighbor distance, and the $\sigma$ 's are the eigenvectors of the Fourier-transformed dynamical matrix divided by the square roots of the appropriate masses. [For example, $\sigma_{x}\left(k \mid-\mathbf{q}, j^{\prime}\right)$ contains a factor of $\left.m_{1}^{-1 / 2}.\right] \mathrm{It}$ is important to note that two phase conventions are used in Born and Huang, one in Chap. V and a second in Chap. VI. In their formula for $P_{\alpha \beta}$ given in Eq. (39.16) the second convention is used. We have used the first, since this is the form in which the eigenvectors from our equations of motion are obtained.

As they stand, Eqs. (3) and (4) are of no practical use since they contain too many unknown constants. The number can be reduced by making what is, at this stage, only a plausible assumption: namely, that the polarizability is affected only by central displacements of the nearest neighbors (i.e., changes in bond lengths). If this is done, it is found that $b=c=d_{1}$ and $e=f=g=0$.

${ }^{14}$ M. Born and M. Bradburn, Proc. Roy. Soc. (London) A241, 105 (1948).
This second result is extremely important, because it means that $P_{y^{2}}=0$.

Now for the scattering geometry we are considering, it follows from Eqs. (49.1) and (49.2) of Born and Huang that the scattering intensity of the polarized component $I_{1 I}\left(\mathbf{q} ; j j^{\prime}\right)$ for a given combination is given by

$$
I_{11}\left(\mathbf{q}, j j^{\prime}\right)=A\left[\frac{P_{z z}\left(\mathbf{q}, j ;-\mathbf{q}, j^{\prime}\right)}{\left(\omega_{\mathrm{q}, j} \omega_{-q, j^{\prime}}\right)^{1 / 2}}\right]^{2},
$$

while the depolarized intensity is given by

$$
I_{\perp}\left(\mathbf{q} ; j j^{\prime}\right)=A\left[\frac{P_{y z}\left(\mathbf{q}, j ;-\mathbf{q}, j^{\prime}\right)}{\left(\omega_{\mathbf{q}, j} \omega_{-\mathbf{q}, j^{\prime}}\right)^{1 / 2}}\right]^{2},
$$

where the $\omega$ 's are the phonon frequencies, and $A$ is some constant, proportional to the incident intensity.

We can now see that, since the observed spectrum is effectively $100 \%$ polarized, then one can infer that $P_{y z} \simeq 0$. Thus, the "central polarizability" assumption is strongly supported by the experimental results, and we may therefore be able to predict the form of the observed polarized spectrum using only three parameters : $a, b$, and $d_{2}$. However, since we only need to derive relative intensities, we have only two disposable con- 
Fig. 4. Predicted polarized spectra for the RI model using the two polarizability options, 1 and 2, described in the text. (a) Predicted intensities, in arbitrary units on a linear scale, of the $0^{\circ} \mathrm{K}$ Stokes spectra plotted against the $0^{\circ} \mathrm{K}$ combined dispersion curves. The correspondence between the various peaks in the spectrum and critical points, where this exists, is indicated by labels $a, b$, etc., on both the spectra and on the dispersion curves. (b) Intensities in arbitrary units of the $300^{\circ} \mathrm{K}$ Stokes and anti-Stokes spectra for both options for the RI model. Experimental features are labeled $A$, $B$, etc. [Note that in (a) and (b) the zero of intensity for variation 2 is displaced vertically with respect to variation 1 . Also, although both variations are shown with a common (arbitrary) scale of intensity, we are not predicting the relative intensity except that it is constant throughout the spectrum.]
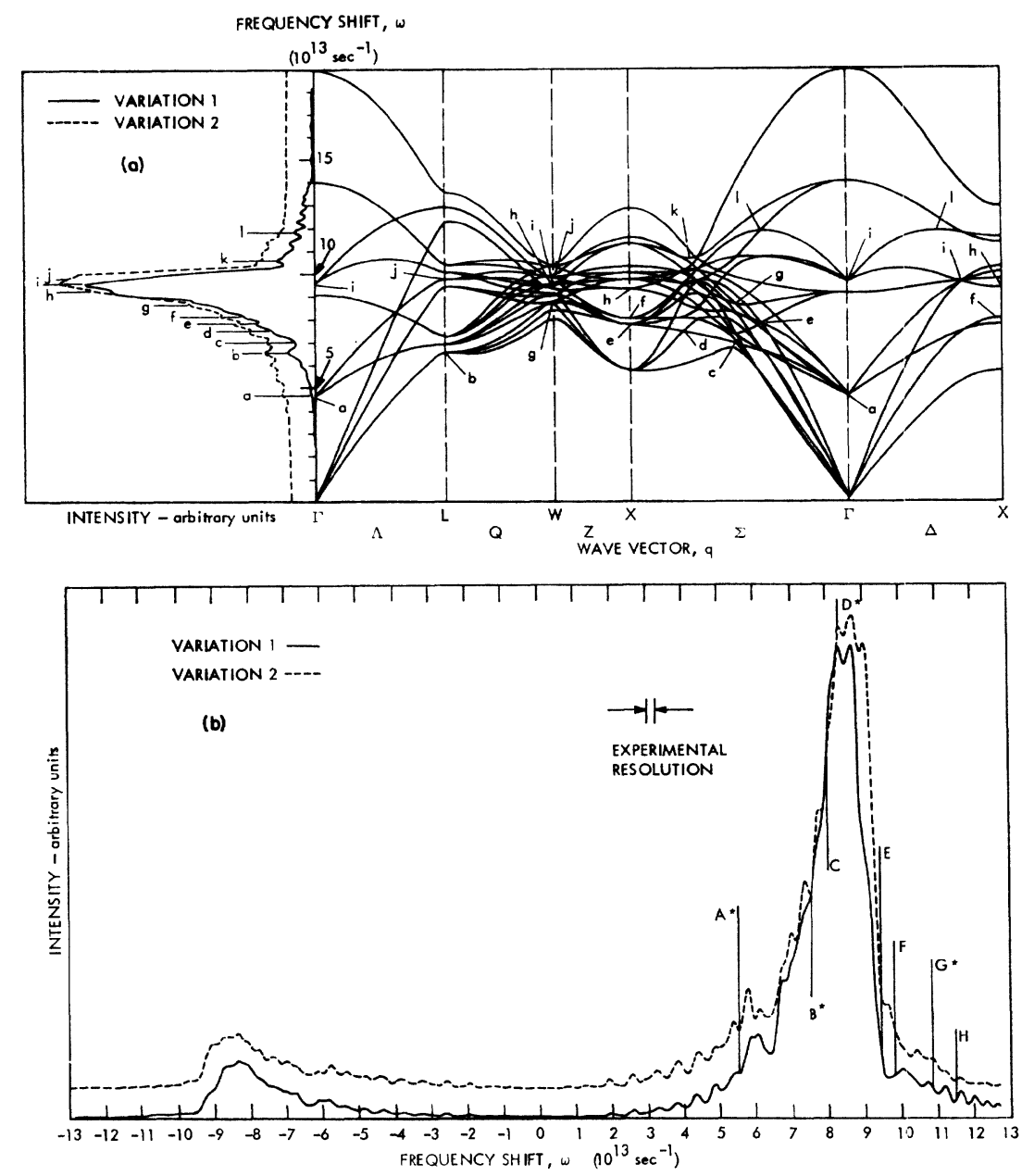

stants: $b / a$ and $d_{2} / a$. What we now propose to show is that, within reasonable limits, the calculated spectrum, at least as far as the Stokes and anti-Stokes components are concerned, is relatively insensitive to these ratios. However, we shall also find that the calculated difference bands are much more sensitive to the ratio $d_{2} / a$.

The intensity to be calculated is given by the righthand side of Eq. (1), except that each sample combination must be weighted by the appropriate value of

$$
I_{11}\left(\mathbf{q} ; j j^{\prime}\right) \text {, }
$$

derived from Eqs. (3) and (5). As in the case of the simple combined density-of-states calculation, it is expedient to calculate the Stokes and anti-Stokes components simultaneously, but to display the difference components separately. The reason for this is that, while the densities of states for Stokes and anti-Stokes processes are mirror images, difference processes are determined by a completely distinct density of states, and it turns out that they display a completely different model dependence from the Stokes and anti-Stokes lines.
Because we have no real a priori knowledge of the magnitudes of the ratios $c / a$ and $d_{2} / a$, we have made calculations for what seem to be two reasonable extreme sets of values that should effectively bracket the true values. At one extreme (variation 1) we assume that $c=d_{2}=0$ and at the other extreme that $a=d_{2}$ and $c / a=-2 \rho / r_{0}$, where $\rho$ is the Born-Mayer screening radius for the overlap repulsive potential.

These choices seem to be reasonable, since in the first case we are almost certainly underestimating the ratio of $d_{2} / a$, while in the second we are probably overestimating it. Moreover, it is unlikely that $d_{2}$ and $a$ will have opposite signs. Considering the choice of $c / a$, again the first option probably represents an underestimate while the second option is plausible, since $c$ represents the effect of bond shearing, which is expected to be smaller than that of bond stretching and of opposite sign.

In Fig. 4 the calculated spectra are shown for variations 1 and 2 for the RI model computed for both 0 and $300^{\circ} \mathrm{K}$. At very low temperatures there exists only a Stokes spectrum, which has been plotted together with the combined dispersion curves along the principal 


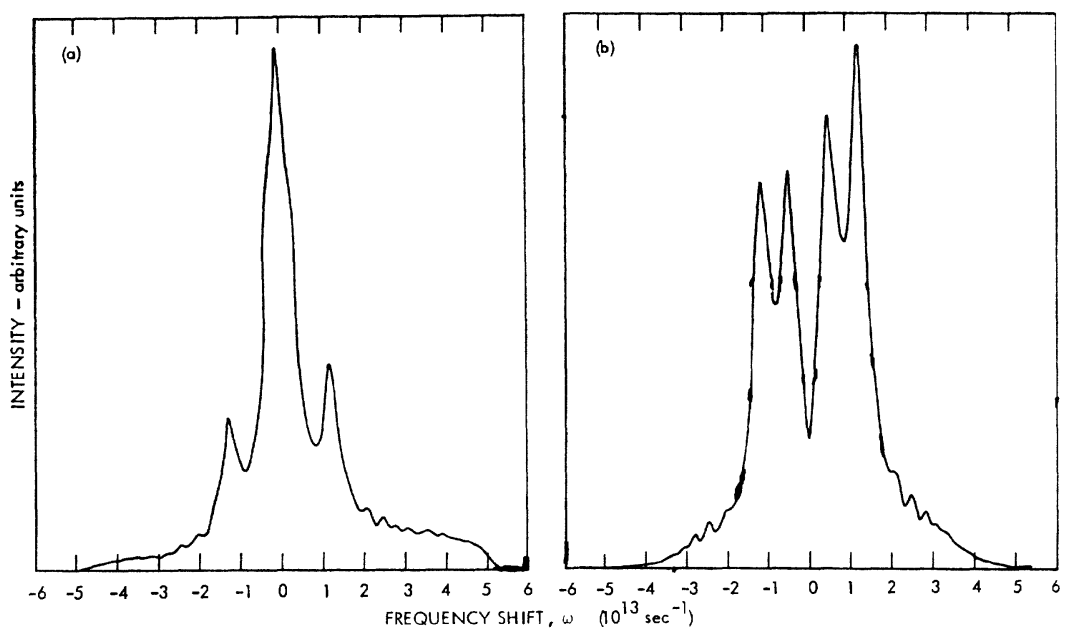

Fig. 5. Polarized $300^{\circ} \mathrm{K}$ difference bands predicted for the RI model including the effects of variations 1 and 2 in the polarizability tensor. (a) Variation 1. (b) Variation 2. (Note that the intensity scales are linear but differ in various figures so that the results can be conveniently displayed.)

symmetry directions. For the room-temperature case, the Stokes and anti-Stokes combination spectra are given, since we wish to discuss the difference bands separately. In Fig. 5, the difference spectra corresponding to $300^{\circ} \mathrm{K}$ are shown. Corresponding results for the DDNNN model are given in Figs. 6 and 7 . Throughout these calculations, the same sample of $\mathbf{q}$ vectors and the same Gaussian smoothing technique have been used as for the combined density-of-states calculations.
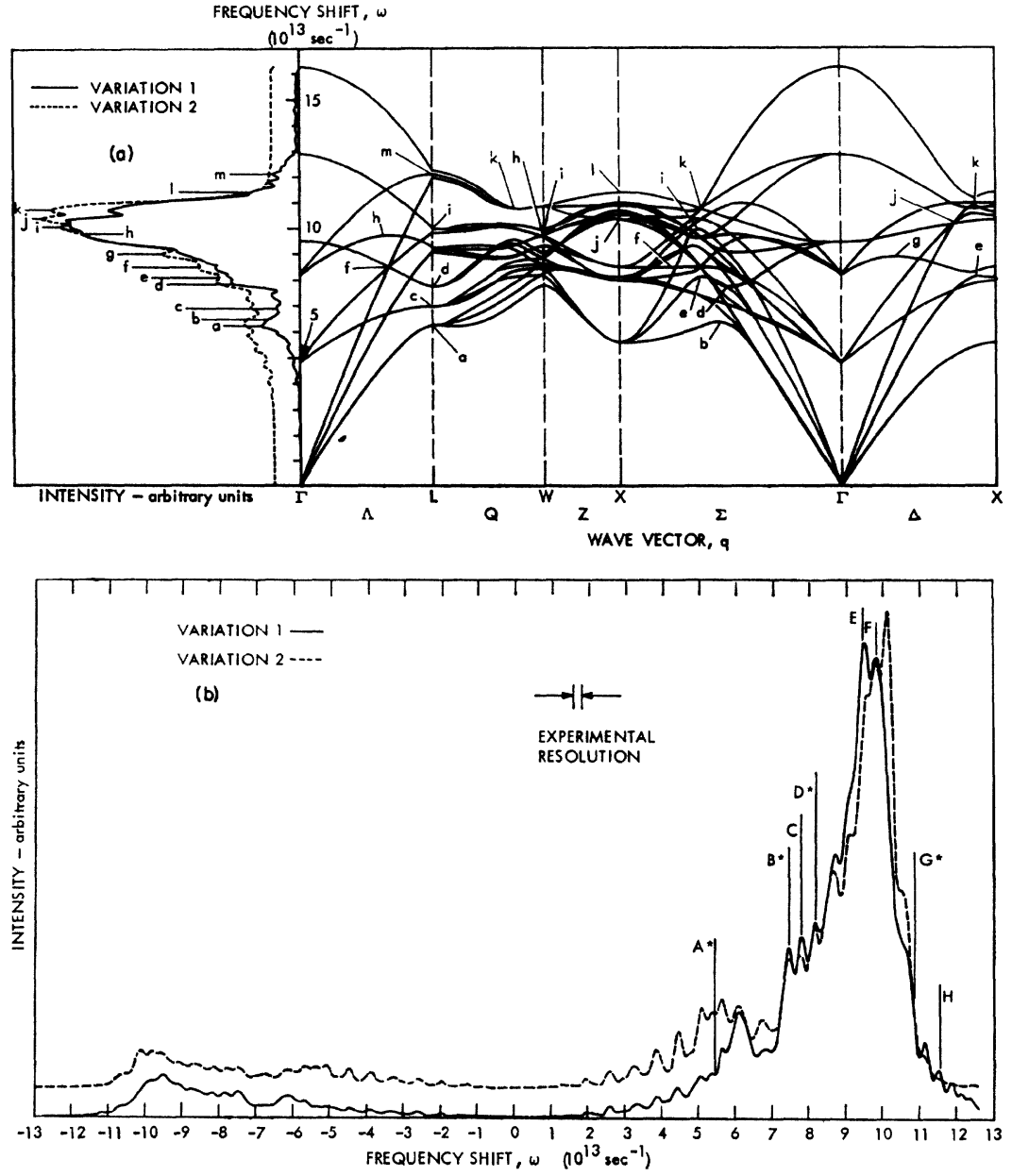

Fig. 6. Predicted polarized spectra for the DDNNN model using the two polarizability options described in the text. (a) Predicted $0^{\circ} \mathrm{K}$ Stokes spectrum versus combined dispersion curves with critical points indicated. (b) Predicted $300^{\circ} \mathrm{K}$ Stokes and antiStokes spectra with the experimental features labeled as in Fig. 4. 
FIg. 7. Polarized $300^{\circ} \mathrm{K}$ difference bands predicted for the DDNNN model including the effects of variations 1 and 2 in the polarizability tensor. (a) Variation 1. (b) Variation 2 .

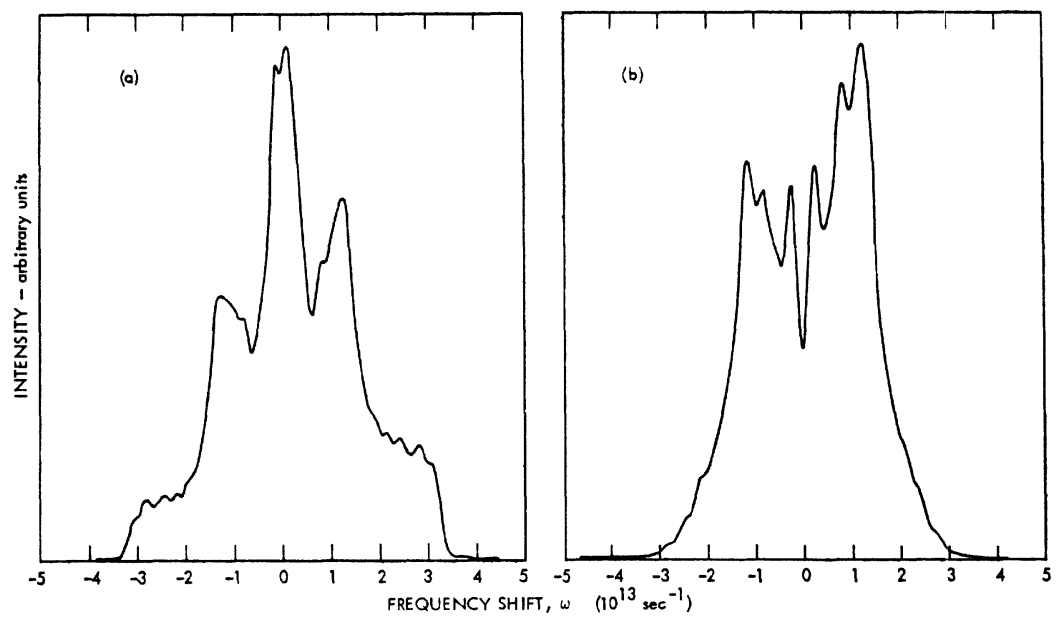

\section{DISCUSSION OF RESULTS}

From a comparison of Figs. 2 and 3 with Figs. 4-7, it is evident that the general effect of including the polarizabilities is a marked suppression of subsidiary structure. In particular, there is one important and striking improvement: namely, the strong sharp peaks near the exciting line in the $300^{\circ} \mathrm{K}$ densities of states have been removed. This is satisfying because these are unphysical and have their origin in the very large occupation numbers of the low-frequency acoustic phonons, leading to a gross overweighting of the corresponding two-phonon processes. When the polarizabilities are included, this overweighting is eliminated by the fact that the intensities tend to zero as $q \rightarrow 0$ for pairs of acoustic phonons. Physically, this corresponds to the correct limiting behavior, since a uniform translation of the crystal is obviously not Raman active to any order. These results are also relevant to our earlier work on the $300^{\circ} \mathrm{K}$ Raman spectra of the cesium halides, ${ }^{8}$ where we obtained similar peaks for the same reason.

Apart from the removal of this anomaly, the effects of allowing for the variations of the Raman polarizability tensor are extremely striking. This can be seen in detail in Figs. 4 and 6, where the positions of the various observed features are shown on both the RI and the DDNNN-computed Stokes spectra appropriate to $300^{\circ} \mathrm{K}$. It can be seen that the RI spectra for both variations 1 and 2 are in very bad agreement with experiment. Almost all the computed features, in particular the main peak, occur at frequencies about $10 \%$ lower than the corresponding experimental features. Moreover, the main computed peak is significantly sharper than that in the observed spectrum. However, when we refer to the computed DDNNN spectra, the agreement between theory and experiment is remarkable. For variation 1 we can justifiably say that within the limits of accuracy imposed by resolution on the experimental spectrum, the agreement between the positions of experimental and theoretical features is exact. However, the relative intensities of the two components of the main peak, $E$ and $F$, are reversed in the theoretical spectrum; the former being slightly stronger. This is the only definite discrepancy between theory and experiment. The appearance of subsidiary structure in the theoretical curve which is apparently absent from the experimental spectrum is of no significance at present, since the experimental resolution is too broad to reveal these features which could be genuinely present.

With regard to the spectrum predicted by variation 2 , one can see from Fig. 6 that, except on a very fine scale, it is almost the same as that predicted by variation 1 . The only significant difference is in the structure of the main peak. Here the relative intensities of the high- and low-frequency components are in the correct order compared with the experimental peak, but the whole peak occurs at slightly too high a frequency. However, this discrepancy is insignificant at present since it is scarcely larger than the uncertainty in the positions of the experimental features.

Thus, we can say that both variations 1 and 2 for the DDNNN model reproduce the observed spectrum with remarkable precision. In fact, as far as we know, nothing approaching the present agreement between theory and experiment has yet been achieved in any other work on the second-order Raman spectra of other crystals. To some extent this is probably due to the marked insensitivity of the computed spectra to the relative magnitudes of the polarizability tensor components for the particular case of $\mathrm{NaF}$. We have recently completed a corresponding investigation ${ }^{15}$ of $\mathrm{CsF}$ and find very large differences between the results of variations 1 and 2. Finally, for $\mathrm{NaF}$, since we cannot rule out the existence of a very weak depolarized spectrum, we have tried using a set of parameters $a, b$, etc., for the polarized spectrum which are not subject to "central" constraints. This is a mixture of variations 1 and 2 with only $a$ and $b$ nonzero. The resulting spectrum

\footnotetext{
${ }^{15}$ A. M. Karo and J. R. Hardy, Phys. Rev. 168, 1054 (1968).
} 
is so similar to variation 1 that it is not reproduced. The only significant difference is that the main peak $(E, F)$ resembles the experimental peak even more closely in that $F$ is the stronger component, with $E$ appearing as a slightly weaker low-frequency shoulder.

For the difference bands, we can only present the theoretical results, since there are no experimental data. It can be seen that for each model, variations 1 and 2 lead to strikingly different results. This in itself fully justifies separate presentation of these data. Obviously, experimental observation of these difference spectra would provide a very useful way of fixing the relative magnitudes of the parameters $a, b$, etc., in the polarizability tensor. This problem could also be usefully examined by more precise measurements on the $300^{\circ} \mathrm{K}$ Stokes spectra. In addition, it would be interesting to have low-temperature data available to compare with our predicted spectra; conceivably these would show more fine detail. In all cases, it is obviously desirable that the intensities be measured by some strictly linear recorder. 